\title{
Experiencias de reducción de daños relacionados con las drogas en Buenos Aires
}

\author{
Cymerman, P.; Ereñú, N.; Goltzman, P.; Faraone, S.; Rojas, E.; Rossi, D.; Sánchez, A.; Touzé, G.; \\ VÁZQUeZ, S.; VILA M.
}

\section{Resumen:}

En la Argentina se observa un alto porcentaje de casos de SIDA asociados con el uso compartido del equipo de inyección (41\%) y con las prácticas sexuales sin protección.

Tomando en consideración que los usuarios de drogas inyectables frecuentan las farmacias para la compra de jeringas, la asociación civil Intercambios puso en marcha el proyecto "La farmacia como ámbito de prevención del VIH/SIDA". Se capacitó personal de farmacias de la ciudad de Buenos Aires y se puso en marcha un proyecto con 24 farmacias para el desarrollo de acciones de reducción de los riesgos de infección por VIH en usuarios de drogas inyectables.

En otro proyecto se estudió el perfil de los usuarios de drogas en Buenos Aires mediante la realización de 60 entrevistas a informantes clave y 60 entrevistas en profundidad a usuarios de drogas inyectables y se desarrolló un proyecto piloto de reducción de los riesgos de infección por VIH en la periferia de Buenos Aires. Es el primer proyecto comunitario orientado a usuarios de drogas inyectables que permite acumular experiencia en el empleo de estrategias de reducción de daños en la Argentina.

Palabras clave: reducción de daños, farmacias, Sida, intercambio de jeringas, Argentina, drogadictos por vía inyectable.

\section{Summary:}

In Argentina a high percentage of AIDS cases has been observed associated with the shared use of the injection apparatus $(41 \%)$ and with unprotected sexual relations.

Taking into consideration that users of injectable drugs frequent chemists in order to buy syringes, the civil association Intercambios carried out the campaign "The chemist as a preventive sphere of HIV/AIDS". Personnel from the Buenos Aires city chemists were given training and a project was organised with 24 chemists to take actions leading to the reduction of the risks of infection by HIV in users of injectable drugs.

Another project studied the profile of drug users in Buenos Aires by carrying out 60 interviews of key informants and 60 indepth interviews of intravenous drug users, and a pilot project for the reduction of the risk of HIV infection was carried out on the outskirts of Buenos Aires.

It is the first community project orientated at intravenous drug users which allows the accumulation of experiences in the use of harm reduction strategies in Argentina.

Keywords: harm reduction, pharmacy, Aids, needle exchange, Argentine, intravenous drug users

\section{INTRODUCCIÓN}

$\mathbf{L}$ a magnitud de los problemas de salud pública entre los usuarios de drogas inyectables, tales - como la transmisión del VIH, la hepatitis B y C, ha jugado un papel fundamental para revisar los enfoques desde los que se aborda la práctica del uso de drogas. El marco conceptual que guía y unifica la mayoría de los programas desarrollados desde la perspectiva de la Salud Pública, se conoce comúnmente como "reducción de daños".

En la Argentina, un número muy importante de personas que usan drogas inyectables se han visto afectadas por distintas enfermedades relacionadas con su vía de administración. Es así como se observa un alto porcentaje de casos de SIDA (41\%) asociados con el uso compartido del equipo de inyección y con las prácticas sexuales sin protección (1). Además, muchos usuarios de drogas tienen dificultades para acceder a los servicios de salud.

Es en este contexto que la Asociación Civil Intercambios ha desarrollado un proyecto de prevención del VIH/SIDA en usuarios de drogas a los que llega por intermedio de las farmacias y un proyecto de investigación e intervención orientado a la población de usuarios de drogas inyectables de una zona de la periferia de la Ciudad de Buenos Aires. 


\section{LAS FARMACIASY EL VIH/SIDA}

En la Argentina, el sector farmacéutico ha corroborado mediante la firma de un convenio, suscrito el 1 de diciembre de 1997 entre el Ministerio de Salud y Acción Social y la Confederación Farmacéutica Argentina su voluntad de colaborar en tareas de información y educación a fin de evitar los riesgos que facilitan la infección por VIH y sus consecuencias (2).

En la ciudad de Buenos Aires existen 1600 farmacias con atención al público. Estas se nuclean en 4 instituciones principales: la Asociación de Empleados de Farmacias, la Asociación de Farmacias Mutuales y Sindicales de la República Argentina, la Cámara Argentina de Farmacias y el Colegio de Farmacéuticos y Bioquímicos de la Capital Federal.

En este contexto, la Asociación Civil Intercambios inició en abril de 1998, el proyecto "La farmacia como ámbito de prevención del VIH/SIDA en usuarios de drogas", que fue aprobado en el concurso de proyectos del Programa Lusida del Ministerio de Salud y Acción Social de la Nación, con los siguientes objetivos:

- Sensibilizar al personal de farmacia acerca de su rol como actor de primera línea en la prevención del VIH/SIDA.

- Capacitar a farmacéuticos y auxiliares de farmacia como agentes de prevención del VIH/SIDA, en especial en usuarios de drogas inyectables.

- Desarrollar e implementar en farmacias de la ciudad de Buenos Aires, acciones de reducción de los riesgos de infección por VIH en usuarios de drogas inyectables.

El proyecto contó desde su inicio con las participación y el apoyo activo de las cuatro asociaciones vinculadas a la actividad farmacéutica en la ciudad de Buenos Aires. Durante la primera etapa, se realizó la indagación sobre las características de funcionamiento y actividades de cada institución.

Asimismo se acordó la publicación en los medios de comunicación de las asociaciones, de artículos de difusión acerca de la problemática del VIH/SIDA y el uso de drogas. Se envió información sobre el proyecto a las 1600 farmacias de la ciudad de Buenos Aires. Para ello se editó un tríptico de presentación que fue distribuido a través de los canales de las instituciones y se organizaron los cursos taller para la capacitación del personal de farmacias.

Se realizaron grupos focales con farmacéuticos y auxiliares de farmacia para conocer sus necesidades de capacitación y adecuar las estrategias didácticas, así como para apoyar el diseño y validación de material educativo sobre prevención del VIH/SIDA específicamente dirigido a personal de farmacias.

\section{Capacitación del personal farmacéutico}

Se desarrollaron 4 cursos-taller dirigidos a personal de farmacias. Se realizaron en las sedes de las 4 instituciones antes mencionadas, con la participación de 96 personas.

El objetivo de esta capacitación fue mejorar el nivel de conocimiento acerca de la problemática VIH/SIDA y uso de drogas, mejorar las destrezas de comunicación; informar sobre los recursos existentes y brindar un apoyo técnico a este proceso.

Al iniciar cada uno de los cursos taller, realizamos una encuesta entre los participantes para ver cuales eran sus expectativas y la realidad en que desarrollaban su tareas.

Las expectativas más frecuentemente mencionadas fueron:

- Obtener información actualizada

- Capacitarse para realizar trabajos preventivos en farmacias

- Referencias directas a usuarios de drogas.

- Referencias directas al VIH/SIDA

Más del 50\% reconocía la presencia de usuarios de drogas en los locales de farmacia.

\section{CONTACTO CON USUARIOS}

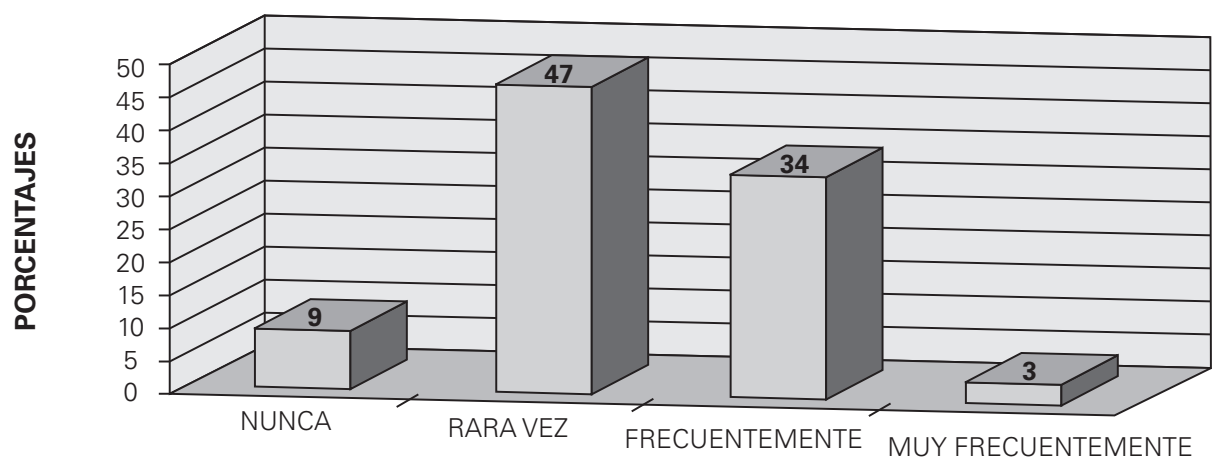


En el diálogo con los farmacéuticos se caracterizaron dos tipos de situaciones: la demanda de jeringas y la demanda de productos farmacéuticos con recetas falsificadas. Respecto de estos medicamentos, hubo coincidencia en la decisión de no venderlos.

Respecto de la venta de jeringas a usuarios de drogas, sin embargo, se planteó una diferente posición: quienes las vendían argumentaron que "sino lo haces, los obligas a compartirlas". No obstante, algunos opinaron que por razones comerciales era mejor no venderlas argumentando: "no quiero a esa gente como clientes y si le vendes a uno, se te llena el local."

Durante el desarrollo de los cursos se entregó un cuadernillo que resumía el contenido de los cursos taller. "Orientación para el Trabajo en Farmacias" (3).

Las personas que concurrieron a la capacitación eran mayoritariamente farmacéuticos, directores técnicos de las farmacias, hubo también auxiliares y en menor medida empleados, encargados y gerentes. Un $82 \%$ de ellos no había recibido capacitación previa sobre el tema, pero todos consideraron que es posible realizar prevención del VIH/SIDA en farmacias.

Luego de finalizados los cursos y a partir de una invitación nuestra, muchos manifestaron que deseaban continuar en contacto con el proyecto. Ya sea incluyendo a su farmacia en las actividades a desarrollarse, integrando un grupo de trabajo permanente, recibiendo visitas periódicas de los operadores del proyecto, participando en reuniones o recibiendo información por los medios de difusión de las asociaciones.

\section{Prevención en las farmacias}

Se pre-seleccionaron 35 farmacias, las que fueron visitadas con el objetivo de observar sus características, su ubicación, entrevistar a los contactos y conocer al resto del personal y a los gerentes y/o propietarios.

Los criterios de selección incluyeron: a) interés y disponibilidad del personal de la farmacia; b) ubicación geográfica (cobertura de diversas zonas de la ciudad y cercanía con áreas de probable uso de drogas); c) reconocimiento de concurrencia de usuarios de drogas al local. Finalmente se seleccionaron 24 farmacias que fueron incluidas activamente en el Proyecto.

Las acciones preventivas en las farmacias se lanzaron con una actividad conjunta para el 1 de diciembre, Día mundial de lucha contra el sida. En esa ocasión se desarrollaron las siguientes actividades:

- Preparación de vidrieras alusivas.

- Distribución de folleto preventivo.

- Distribución de preservativos.

- Entrega de cintas rojas.
Durante ese día, se recorrieron las 23 farmacias en las que se estaban realizando actividades. Se calcula que el $1^{\circ}$ de Diciembre se distribuyeron 10.000 folletos de información preventiva y 6.000 preservativos en las farmacias participantes, las que continuaron con las actividades los días subsiguientes. En todas las farmacias, se armaron vidrieras alusivas, que continuaron así, al menos durante toda la semana. Varios medios de comunicación difundieron estas actividades.

Con posterioridad se iniciaron las acciones específicamente dirigidas a usuarios de drogas, que consistieron en la distribución de material preventivo específico dirigido a usuarios de drogas inyectables, en el momento de la venta de jeringas en las farmacias participantes del proyecto.

Para identificar a estas farmacias, se diseñó un afiche. El objetivo era doble: la identificación de la farmacia y el mensaje preventivo que contiene.

Se diseñó un desplegable, que fue validado en 3 grupos focales, uno con usuarios de drogas en tratamiento, otro con usuarios de drogas activos y un tercero con personal de farmacias. Este desplegable presentaba el siguiente contenido:

- TAPA: Para cuidarte del sida se usa una vez y se tira. Con dibujos de una jeringa y un preservativo.

- MENSAJES PREVENTIVOS:

Riesgos del uso inyectable de drogas.

No compartir agujas ni el equipo de inyección.

Limpieza del equipo de inyección.

Descarte del material de inyección.

Uso correcto del preservativo.

- CONTRATAPA: Si tenés dudas en la farmacia te pueden ayudar.

El criterio de entrega es hacerlo a todas las personas que compran jeringas en la farmacia, a fin de no reforzar estereotipos estigmatizantes.

Asimismo, a partir de la entrega de material estimulamos que el farmacéutico esté disponible para las demandas que pudieran surgir. Que el farmacéutico y la farmacia sean un lugar de referencia donde las personas puedan encontrar una escucha para evacuar sus dudas y/o contar con la posibilidad de conocer los recursos con que cuenta la comunidad.

Entre los meses de diciembre y mayo próximo pasado, se han distribuido alrededor de 4.000 desplegables y se han atendido aproximadamente 200 consultas en demanda de información: lugares de tratamiento, medidas de bioseguridad, solicitud de charlas en clubes y escuelas del barrio.

En abril de 1999 y como cierre de la primera etapa del proyecto, se realizaron las Primeras Jornadas sobre Prevención del VIH/SIDA en la farmacia. Las mismas se propusieron como un espacio de encuentro entre el personal de las farmacias y los equipos que trabajan 
con las problemáticas del VIH/SIDA y el uso de drogas. Participaron más de 100 personas y contaron con la participación de personalidades del ámbito académico e invitados extranjeros. Se presentó el video que reseña el proceso desarrollado y que fue distribuido en el medio farmacéutico de todo el país.

En términos del impacto sobre la población objetivo (usuarios de drogas inyectables), se estima que éste ha resultado de relativo alcance en términos cuantitativos, situación prevista en la propuesta del proyecto, dado su carácter de experiencia piloto. No obstante, en términos cualitativos se estima que el proyecto ha comprobado la eficacia de las intervenciones propuestas y que, fundamentalmente ha creado las condiciones para una aplicación ampliada, a efectos de lograr el impacto esperado.

En los destinatarios directos (personal de farmacias) se han evidenciado modificaciones tanto en sus representaciones como en sus prácticas, incorporando contenidos de prevención a su actividad cotidiana.

Por otra parte, y como resultado no buscado pero de valor estratégico, se advierte el carácter innovador de la experiencia en el contexto latinoamericano, ya que sólo en Brasil se encuentran iniciativas similares.

\section{INVESTIGACIÓN E INTERVENCIÓN CON UDIS}

\section{Objetivos y Metodología del estudio}

El Grupo Temático de ONUSIDA de la Argentina promovió un proyecto de investigación e intervención ejecutado por la Asociación Civil Intercambios y financiado por ONUSIDA, desde septiembre de 1998.

Sus objetivos se orientaron a conocer el perfil de los usuarios de drogas en Buenos Aires y a desarrollar e implementar un proyecto piloto de reducción de los riesgos de infección por VIH en usuarios de drogas de esta ciudad.

Se adoptó la metodología de Evaluación y Respuesta Rápida, con el uso de las “Guías para la Evaluación Rápida y Desarrollo de Respuestas en el Uso de Drogas por Vía Inyectable (UDI-ERR) y en el Uso de Sustancias y la Conducta Sexual (SEX-ERR)", elaboradas para el Programa de Abuso de Sustancias de la Organización Mundial de la Salud (PSAMHO) y por el Centre for Research on Drugs and Health Behaviour, Department of Social Sciences and Medicine, Imperial College School of Medicine (Londres, Reino Unido) (4).

\section{Información existente}

Se obtuvo información de fuentes secundarias proveniente de más de 50 estudios publicados; de pro- yectos de investigación; planes, programas, informes, materiales de difusión y datos estadísticos de organizaciones gubernamentales y no gubernamentales; se analizaron leyes, ordenanzas, resoluciones y material periodístico.

Se dio prioridad a los siguientes aspectos:

- Contexto político del uso de drogas

- Patrones socioculturales del uso de drogas

- Datos nacionales sobre tasas y tendencias del uso y problemas relacionados; y datos sobre las características de los usuarios de drogas;

- Los problemas de salud asociados con el uso de drogas, con especial énfasis en la infección por $\mathrm{VIH}$;

- El uso de los servicios de salud por parte de las personas con problemas relacionados con las drogas.

Del análisis de los datos recolectados surgen los siguientes vacíos en la información:

- Escasa actualización de los datos existentes. Muchos de los trabajos a los que accedimos eran de la primera mitad de esta década. Estos datos eran interesantes para ubicar en contexto histórico la problemática pero poco nos decían de la situación actual del uso de drogas.

- Escasa producción de datos acerca de la vía de consumo. Los trabajos consultados en general ponen el énfasis en el tipo de sustancia consumida y no en la vía de uso.

- En casi la totalidad de las investigaciones, se hace referencia a población que consultó en centros de tratamiento o población internada, pero encontramos sólo dos trabajos, que indagan información de población no vinculada con instituciones de tratamiento en el momento de realización de las investigaciones.

- Por último, otro aspecto a considerar es el referido al desconocimiento de datos globales acerca de la población que vive con VIH y en forma particular, la incidencia del consumo de drogas inyectables y su relación con el VIH. Los escasos datos registrados en este sentido también refieren siempre a población que consultó en algún centro asistencial.

\section{Entrevistas a informantes clave}

Con el fin de construir el perfil del uso de drogas inyectable en Buenos Aires, se realizaron 60 entrevistas a informantes clave, que arrojaron la siguiente información (5):

- Aumento en el uso de la cocaína en Buenos Aires en la última década que se consume por distintas vías, principalmente por aspiración. 
- La cocaína es la droga preferida para uso inyectable.

- Hace una década los usuarios de drogas inyectables (UDIs) comunicaban más su práctica en los ámbitos sanitarios sin ocultarla como se observa actualmente.

- No se identifican lugares donde los UDIs desarrollen su práctica en presencia de otras personas que no son UDIs.

- El uso inyectable de drogas se percibe como una práctica solitaria o de grupos cerrados.

- Se registra una menor afluencia de UDIs a los servicios de atención.

- Se percibe una menor visibilidad de la práctica inyectable en los más jóvenes.

- Hay una percepción generalizada respecto de la disminución del uso inyectable en Buenos Aires.

Esta información denota que se percibe un aumento del uso de cocaína, pero al mismo tiempo una disminución de la elección de la vía inyectable, lo que pareciera ser un cambio en las prácticas de los últimos años.

\section{Entrevistas en profundidad}

La información ofrecida por los informantes clave, se complementa con la obtenida de las entrevistas en profundidad realizadas a 60 UDIs de Buenos Aires.

La muestra de UDIs fue no probabilística intencional. Se llegó a la población por medio de la técnica de bola de nieve que consiste en pedir a cada sujeto encontrado que presente uno o más sujetos que sean adecuados para el estudio. Este tipo de técnica de recolección de datos es muy empleada para el estudio de poblaciones ocultas.

Los criterios de selección incluyeron varones y mujeres que hicieran uso de drogas inyectables en el último año, que no estuvieran internados en centros de tratamiento por drogas, ni en instituciones carcelarias o manicomios y que habitaran en Buenos Aires.

Luego de realizado el estudio, estaba previsto iniciar intervenciones preventivas en la población UDI, por lo que necesitábamos ubicar a un conjunto de sujetos que tuvieran algún tipo de vinculación entre si. Los UDIs en la Ciudad de Buenos Aires fueron muy difíciles de encontrar y entrevistar y no parecían ser integrantes de redes de consumidores. Fue más sencillo hallar redes visibles de UDIs en el conurbano bonaerense, por lo que conseguimos realizar 38 de las 60 entrevistas en la zona sur, en el partido de Avellaneda, donde localizamos el proyecto. Las condiciones de vida de los entrevistados en esa zona a la que llegamos por intermedio de otros UDIs son muy precarias. El $13.3 \%$ de la población total del Distrito de
Avellaneda vive en hogares con necesidades básicas insatisfechas, de los cuales el 93.58\% se encuentran localizados en villas de emergencia y asentamientos (6).

Si bien los informantes clave nos citaron ejemplos de uso inyectable en todos los sectores sociales, nosotros llegamos a sectores medios y bajos, lo que no significa que sólo en esos ámbitos se de este tipo de práctica.

\section{Caracterización de los UDls de la muestra}

El $85 \%$ de nuestra muestra son varones y el 15\% son mujeres. Tuvimos especial interés en encontrar mujeres UDIs, ya que parece ser una práctica mucho más propia de los varones, las UDIs están mucho más estigmatizadas y también más ocultas.

Los datos obtenidos indican que llegamos a una población que es mayoritariamente masculina, joven, soltera, con bajo nivel de instrucción, con empleos sin calificación y sin relación de dependencia. Muchos de ellos viven en zonas marginales de Buenos Aires y en general no son el principal sostén del hogar.

Respecto del uso de drogas, la edad promedio de inicio en el consumo de drogas en general fue de 15 años, pero la edad promedio de inicio en el consumo de drogas por vía inyectable fue de 18 años. Un 44\% de la población empezó a usar drogas inyectables entre los 17 y los 18 años y un 20\% antes de los 17; mientras que el uso de drogas por otras vías se concentró en un $78 \%$ de los casos entre los 12 y los 16 años. Si bien hay muy pocos estudios en la Argentina que tomen en consideración la vía de administracióna, la tendencia coincidentemente expresada en las investigaciones es la de un inicio en el consumo de drogas ilegales a edad temprana. El $72 \%$ de los casos de inicio se concentra entre los 10 y los 19 años. La droga de inicio más común sería la marihuana y en mucha menor medida, la cocaína y diversos medicamentos.

El $80 \%$ interrumpió espontáneamente el uso de drogas alguna vez. El 56\% refiere haber recurrido a tratamiento para dejar el uso de drogas. Este dato muestra que los que hicieron tratamiento de abstinencia alguna vez, habían vuelto a consumir y no cambiaron de vía. Ello señala la importancia de promover medidas de reducción de daños durante el período en que los sujetos están en tratamiento.

Las drogas más mencionadas que consumieron por diferentes vías fueron cocaína, marihuana, Rohipnol (tranquilizante), ácido lisérgico, anfetaminas y alcohol. Se mencionó un total de 51 sustancias diferentes consumidas por distintas vías.

En el caso de las drogas inyectables las que tuvieron más menciones como las preferidas fueron cocaí- 
na, anfetaminas en forma de sal, morfina, alcohol y Ketalar (anestésico). Prácticamente no hay referencias a consumo de heroína, que es una droga que hasta el presente no ha sido de elección entre los UDIs, los que parecen estar más interesados en los estimulantes. Esta situación es común a la región que incluye a la Argentina, Brasil, Chile, Paraguay y Uruguay. Por otra parte, la práctica de inyectarse alcohol, principalmente vino parece estar relacionada con que se trata de una sustancia muy barata. Sin embargo, creemos que el alcohol usado por diferentes vías, tuvo menos menciones de las que esperábamos, porque no siempre se lo reconoce como droga. El tabaco nunca fue mencionado.

Respecto del uso inyectable la primera vez siempre se inyectaron en compañía de un amigo, un conocido o un familiar, lo que indica la importancia del trabajo preventivo con pares usuarios de drogas.

El $47 \%$ de los entrevistados se había inyectado por última vez en los últimos dos meses.

El 75\% compartió la jeringa alguna vez. El 7\% la comparte actualmente. Hay una tendencia a no compartir el material de inyección que se verifica en la mayoría de los casos de tres años a esta parte.

No ocurre lo mismo con el resto del equipo de inyección. El recipiente donde se prepara la droga y el agua se comparten con más frecuencia que las agujas y jeringas.

Un 93\% de los entrevistados consigue las jeringas en la farmacia.

Los entrevistados tienen más de un lugar del cuerpo en donde se inyectan habitualmente, pero prefieren el antebrazo porque allí están las mejores venas, por comodidad o porque es donde aprendieron a inyectarse. Muchas veces se eligen otros lugares del cuerpo para que no se note la marca.

El $60 \%$ de los entrevistados se inyecta habitualmente en su casa y el $27 \%$ en casa de amigos. Prevalece el ámbito privado como lugar donde se desarrolla la práctica inyectable, pero hay un $10 \%$ que alguna vez lo hizo en la calle. Los entrevistados nombran entre uno y dos lugares donde se inyectan.

El estado de ánimo y tener dinero es lo que más influye en la frecuencia de inyección. Si tienen dinero todos se inyectarían más. Los que se inyectan una vez por semana o más, comparten con más frecuencia el resto del equipo y lo usan como lo reciben.

Un $80 \%$ refiere cambios en su comportamiento sexual, debido al uso de drogas. Este dato nos lleva a la otra información que buscamos en la entrevista: las características de sus prácticas sexuales.

Los entrevistados son mayoritariamente heterosexuales, muchos de ellos mantuvieron relaciones sexuales con UDIs, pero sus parejas no suelen ser UDIs.
El 37\% de los entrevistados intercambió alguna vez sexo por drogas. Aunque sólo el 15\% de la muestra son mujeres, es importante señalar que parece ser más frecuente esa práctica entre ellas. Algunos de los varones que refieren intercambio de sexo por drogas también hablan de prácticas sexuales con personas del mismo sexo.

Cuando preguntamos acerca de las formas de penetración que conocían, la que tuvo más menciones fue la vaginal, luego la anal y sólo un $47 \%$ mencionó la oral, lo que hace pensar que no la reconocen tanto como forma de penetración. El 98\% tiene relaciones vaginales con más frecuencia.

Un $42 \%$ declara no usar preservativos. El uso del preservativo es menor entre los entrevistados que conviven con su pareja. También, se observa un menor uso del preservativo entre los entrevistados que tienen relaciones con una única persona. Un 38\% dice haber tenido problemas con el uso del preservativo.

Una serie de preguntas estaban referidas a problemas de salud y vínculo con el sistema sanitario. Una proporción importante tiene resultado positivo al test del VIH (57\%), menciona Hepatitis C, enfermedades de transmisión sexual o tuberculosis, pero tienen escaso vínculo con el sistema de salud.

Las tres últimas preguntas de la entrevista se referían a su opinión acerca de distribuir mensajes dirigidos a UDIs respecto del cuidado de la salud, qué contenido tendrían esos mensajes y sus sugerencias. Sólo uno de los entrevistados estuvo en desacuerdo con distribuir esa información.

Del análisis de los datos, surge que los UDIs están más ocultos y estigmatizados a partir de la asociación SIDA-Drogas, ello podría explicar la percepción generalizada de disminución del uso inyectable de drogas, que mencionaron los informantes clave. Las mujeres UDIs están más ocultas y más estigmatizadas que los varones.

\section{Intervenciones}

Cuando el estudio finalizó comenzaron intervenciones preventivas en la zona donde se realizó el mayor número de entrevistas.

Dos tipos de intervenciones fueron desarrolladas: una de ellas se localizó en la casa de un usuario de droga, donde muchos otros usuarios de droga concurren semanalmente; la otra, se localizó en una calle donde un usuario de droga va todas las semanas para encontrarse con otros usuarios de droga. En ambos casos se distribuye información preventiva así como jeringas y condones. Actividades preventivas para el resto de la comunidad que vive en el barrio fueron organizadas por los usuarios de droga y el equipo de investigadores. Algunos de esos usuarios de droga 
son ahora parte de Intercambios como operadores del programa.

\section{Recomendaciones}

Del estudio surgieron las siguientes recomendaciones:

- Necesidad de promover medidas de reducción de los daños en los tratamientos por el uso de drogas dado el alto índice de personas que vuelve a usarlas al salir de ellos.

- Necesidad de incorporar a los equipos preventivos usuarios de drogas que puedan transmitir información a otros UDIs.

- Necesidad de hacer accesible información y material descartable a usuarios de drogas inyectables a fin de consolidar la tendencia de no compartir el equipo de inyección.

- Privilegiar el trabajo preventivo en contextos de uso de drogas, para tener mejor acceso a una población que está oculta.

- Mejorar la articulación con el sistema de salud teniendo en cuenta las necesidades de los UDIs.

\section{LA REDUCCIÓN DE DAÑOS EN LA ARGENTINA}

Si bien los autores de estos proyecto trabajamos desde hace más de 5 años en relación a la problemática del VIH/SIDA, el uso de drogas y las estrategias de reducción de daños, en nuestro país esta corriente ha estado ausente del debate político y técnico hasta hace muy poco tiempo (7).

Actualmente creemos que existen algunas circunstancias que facilitan la discusión y aplicación de estrategias de reducción de daños:

- El primer ejemplo es que el proyecto de Farmacias contó con la financiación del Lusida del Ministerio de Salud y Acción Social de la Nación.

- El Programa Nacional de SIDA y el ONUSIDA han apoyado el proyecto de Investigación y de Intervención en UDIs.

- Argentina participó en la creación de la Red Latinoamericana de Reducción de Daños (RELARD). La RELARD tiene como objetivo promover la reducción de daños asociados al uso de drogas, con prioridad dada a la prevención del VIH en el contexto de la salud pública, y el respeto de los derechos humanos en América Latina. Actualmente, la dirección ejecutiva de la RELARD está en la Argentina a cargo de Graciela Touzé.

- Representantes de la Secretaría para la Prevención de la Drogadicción y la Lucha contra el Nar- cotráfico participaron por primera vez en la $X$ Conferencia Internacional de Reducción de Daños Asociados a las drogas, que se realizó en Ginebra en marzo pasado. El actual Secretario declaró recientemente, por primera vez, que la entrega de jeringas nuevas como medida de salud no podría ser considerada un delito según la legislación nacional.

- La Legislatura de la ciudad Autónoma de Buenos Aires está discutiendo una ley de SIDA para la Ciudad en la que se recomienda la inclusión de las medidas de Reducción de Daños.

- Asimismo, en los últimos tiempos, este debate está siendo presentado en numerosos medios de comunicación social.

\section{REFERENCIAS}

(1) Ministerio de Salud y Acción Social - Programa Nacional de Lucha contra los Retrovirus del Humano, SIDA y ETS. Boletín sobre el SIDA en la Argentina, Julio de 1999.

(2) Correo Farmacéutico, Organo de la Confederación Farmacéutica Argentina, No 34, nueva época, Buenos Aires, diciembre de 1997.

(3) Cymerman, P.; Rossi, D.; Sánchez, A.; Touzé, G. y Vila, M. La Farmacia como Ambito de Prevención del VIH/SIDA. Orientaciones para el trabajo en farmacias, editado por Intercambios, Buenos Aires, septiembre de 1998.

(4) Stimson, G. V.; Fitch, C. y Rhodes, T. (Editores) Guía para la Evaluación Rápida y Desarrollo de Respuestas en el Uso de Drogas por Vía Inyectable. Ginebra, Organización Mundial de la Salud - Programa sobre Abuso de Sustancias, 1998.

Stimson, G. V. y Rhodes, T. (Editores) Guía para la Evaluación Rápida y Desarrollo de Respuestas sobre Uso de Sustancias y Conducta Sexual. Ginebra, Organización Mundial de la Salud - Programa sobre Abuso de Sustancias, 1998.

(5) Rossi, D.; Touzé, G.; Goltzman, P.; Cymerman, P.; Faraone, S.; Ereñú, N.; Rojas, E.; Vázquez, S. Improving the knowledge about drug injecting population in Argentina. $10^{\text {th }}$ International Conference on the Reduction of Drug Related Harm, Ginebra, marzo de 1999.

(6) Ministerio de Economía y Obras y Servicios Públicos. Secretaría de Programación Económica. Documento de Trabajo N 4. CEPA, Comité Ejecutivo para el Estudio de la Pobreza en la Argentina. Buenos Aires, Marzo de 1994.

(7) Touzé, G.; Rossi D.; Cymerman P.; Goltzman P.; Lasala G.; Sánchez A. Harm reduction in Argentina : a challenge to NGO's en International Journal of Drug Policy, Volume 10, Issue 1, Amsterdam, 1999. 
